\title{
tic\&société
}

Vol. 15, $\mathbf{N}^{\circ} 1 \mid$ | 1 er semestre $2021 \mid 2021$

Logique algorithmique et reproduction sociétale: les médiations sociales saisies par les algorithmes

\section{Algorithmes de recommandation et culture technique : penser le dialogue entre éducation et design}

Recommendation Algorithms and Technical Culture: A Dialogue Between

Education and Design

Algoritmos de recomendación y cultura técnica : pensar el diálogo entre

educación y diseño

Arnaud CLAES, Victor WIARD, Heidi MERCENIER, Thibault PHILIPPETTE, Marie DUFRASNE, Arnaud BROWET et Raphaël JUNGERS

\section{OpenEdition}

Journals

Édition électronique

URL : https://journals.openedition.org/ticetsociete/5915

DOI : 10.4000/ticetsociete.5915

Éditeur

Association ARTIC

Édition imprimée

Pagination : 127-157

Référence électronique

Arnaud CLAES, Victor WIARD, Heidi MERCENIER, Thibault PHILIPPETTE, Marie DUFRASNE, Arnaud BROWET et Raphaël JUNGERS, «Algorithmes de recommandation et culture technique : penser le dialogue entre éducation et design », tic\&société [En ligne], Vol. 15, N 1 | 1er semestre 2021 | 2021, mis en ligne le 20 mai 2021, consulté le 02 juin 2021. URL : http://journals.openedition.org/ticetsociete/ 5915 ; DOI : https://doi.org/10.4000/ticetsociete.5915 


\title{
Algorithmes de recommandation et culture technique : \\ penser le dialogue entre éducation et design
}

\author{
Arnaud CLAES \\ arnaud.claes@uclouvain.be \\ Victor WIARD \\ victor.wiard@usaintlouis.be \\ Heidi MERCENIER \\ heidi.mercenier@usaintlouis.be \\ Thibault PHILIPPETTE \\ thibault.philippette@uclouvain.be \\ Marie DUFRASNE \\ marie.dufrasne@usaintlouis.be \\ Arnaud BROWET \\ arnaud.browet@uclouvain.be \\ Raphaël JUNGERS \\ raphael.jungers@uclouvain.be
}


Algorithmes de recommandation et culture technique : penser le dialogue entre éducation et design

\section{Algorithmes de recommandation et culture technique : penser le dialogue entre éducation et design}

CLAES Arnaud: chercheur doctorant au sein du Groupe de Recherche en Médiation des Savoirs (GReMS) de l'Université catholique de Louvain (UCLouvain).

WIARD Victor : chercheur post-doctorant au sein du centre de recherche Engage de I'Université Saint-Louis - Bruxelles (USLB).

MERCENIER Heidi : chercheuse post-doctorante au sein du centre de recherche Engage de l'Université Saint-Louis Bruxelles (USL-B).

PHILIPPETTE Thibault: professeur en information et communication, membre du groupe de Recherche en Médiation des Savoirs (GReMS) de I'Université catholique de Louvain (UCLouvain)

DUFRASNE Marie: professeure en information et communication, codirectrice du centre de recherche Engage de l'Université Saint-Louis - Bruxelles (USL-B).

BROWET Arnaud: chercheur post-doctorant au sein de I'Institute for Information and Communication Technologies, Electronics and Applied Mathematics (ICTEAM) de I'Université catholique de Louvain (UCLouvain).

JUNGERS Raphaël : professeur de mathématiques appliquées, membre de l'Institute for Information and Communication Technologies, Electronics and Applied Mathematics (ICTEAM) de I'Université catholique de Louvain (UCLouvain).

Résumé : Pour répondre à la démultiplication de contenus médiatiques accessibles en ligne, de nombreuses plateformes ont aujourd'hui recours à des algorithmes de recommandation pour structurer et hiérarchiser les flux d'information. Ces dispositifs sont critiqués pour l'importance qu'ils accordent à la satisfaction immédiate de l'utilisateur, menaçant la diversité d'opinion accessible au sein d'espaces médiatiques de plus en plus personnalisés. En croisant les résultats de recherches en sciences sociales et en sciences informatiques, cet article examinera la pertinence de la théorie de la " bulle de filtres ». A travers l'analyse de deux cas d'étude portant respectivement sur 


\section{Arnaud CLAES et al.}

les modalités d'appropriation de systèmes de recommandation par des jeunes et sur la modélisation de dispositifs alternatifs, nous examinerons en quoi la mise en dialogue de l'éducation aux médias et du design peut contribuer à renégocier nos rapports avec des technologies dites " intelligentes».

Mots-clés : algorithme, éducation aux médias, design, bulle de filtres, culture technique

\section{Recommendation algorithms and technical culture: a dialogue between education and design}

Abstract: In order to respond to the proliferation of online media content, many platforms now use recommendation algorithms to structure and prioritize information flows. These technologies are criticized for the importance they attach to immediate user satisfaction, threatening the diversity of opinions available within increasingly personalized media spaces. By combining the results of research in the social sciences and computer sciences, this article examines the relevance of the "filter bubble" theory. Through the analysis of two case studies, one on how young people appropriate recommendation systems and the other on modelling alternative approaches, we will examine how the dialogue between media education and algorithm design can help us rethink our relations with so-called "intelligent" technologies.

Keywords: Algorithm, Media literacy, Design, Filter bubble, Technical Culture

\section{Algoritmos de recomendación y cultura técnica : pensar el diálogo entre educación y diseño}

Resumen: Muchas plataformas utilizan algoritmos de recomendación para estructurar y jerarquizar los flujos de información y así responder a la multiplicación de contenidos mediáticos accesibles en línea. Estos dispositivos son criticados por la importancia que otorgan a la satisfacción inmediata del usuario, amenazando así la diversidad de opiniones accesibles en espacios mediáticos cada vez más personalizados. Combinando los resultados de investigaciones en ciencias sociales y en informática, este artículo analiza la relevancia de la teoría de la "burbuja de filtros". Mediante el análisis de dos estudios de caso sobre las modalidades de apropiación de los sistemas de recomendación por parte de los jóvenes y sobre la modelización de dispositivos alternativos, se analiza cómo el 
Algorithmes de recommandation et culture technique : penser le dialogue entre éducation et design

diálogo entre la educación mediática y el diseño pueden nontribuir a renegociar nuestras relaciones con las tecnologías denominadas "inteligentes".

Palabras clave: Algoritmo, Educación mediática, Diseño, Burbuja de filtros, Cultura técnica.

Remerciements : Nous tenons à remercier nos partenaires de la RTBF et de la plateforme Auvio pour l'accès anonyme aux données ayant permis les analyses de la deuxième étude de cas. Nous remercions également les étudiant(e)s qui ont concédé le droit à réutiliser les représentations graphiques utiles à la compréhension des modélisations algorithmiques.

Le projet de recherche dont est issu ce travail est financé par l'organisation régionale bruxelloise pour la recherche et l'innovation (Innoviris) et son programme Anticipate, sous la référence [2018-ANTICIPATE-24]. 


\section{Arnaud CLAES et al.}

Face à l'usage croissant par des plateformes numériques de systèmes automatisés de recommandation d'information, des critiques se sont élevées pour dénoncer les risques d'isolement cognitif induits par l'usage de ces outils. La personnalisation des contenus recommandés sur la base des comportements de l'utilisateur provoquerait la raréfaction d'informations contraires aux opinions de l'usager facilitant ainsi la création de « chambres d'écho " (Sunstein, 2009) ou de "bulles de filtres " (Pariser, 2011). L'utilisateur serait progressivement dirigé vers des contenus maximisant son temps d'utilisation et sa satisfaction au détriment de la diversité des opinions rencontrées (Yeung, 2017). Toutefois, plusieurs études nous invitent à remettre en question la validité de cette théorie (Moeller et Helberger, 2018; Mutz et Young, 2011 ; Zuiderveen Borgesius et al., 2016).

Ce travail propose d'approfondir cette critique de façon à l'opérationnaliser et à l'appliquer à deux cas d'études. Tandis que le premier traite des usages gravitant autour de ces dispositifs à travers une perspective sociologique, le second discute de modalités de recommandation alternatives en partant de modélisations mathématiques de la notion de bulle de filtres. Sur la base de ces deux cas d'étude, nous tentons de souligner les mérites d'une approche écologique de nos écosystèmes informationnels capable de croiser simultanément des questions d'éducation et de conception informatique.

\section{1 État de l'art sur la problématique de chambre d'écho et de bulle de filtres}

Bien que le terme ne soit jamais clairement défini, la notion de chambre d'écho est notamment mobilisée par Sunstein pour désigner le risque d'isolement informationnel causé par notre tendance, consciente ou non, à favoriser des informations alignées sur nos opinions et intérêts personnels (Sunstein, 2009). Si cette notion est devenue populaire avec l'évolution de notre environnement médiatique, elle peut cependant être rattachée à une littérature plus ancienne portant sur le concept similaire d'homophilie ou d'exposition sélective dans la littérature anglophone (Sears et Freedman, 1967).

Pariser (2011) emploie quelques années plus tard le terme de bulle de filtres pour décrire une situation d'isolement similaire qui serait amplifiée sur Internet et les réseaux sociaux par la présence d'algorithmes de recommandation. À l'instar de celui de chambre d'écho, le concept de bulle de filtres n'est que faiblement formalisé dans la littérature et les deux termes sont parfois mobilisés de façon interchangeable (Bruns, 2019). Pour les besoins de cet article, nous utilisons la notion de bulle de filtres pour désigner un phénomène d'emballement mutuel entre 
Algorithmes de recommandation et culture technique : penser le dialogue entre éducation et design

un mécanisme de filtrage automatisé et un biais d'homophilie. Ces systèmes de filtrage de l'information se basent à la fois sur les similarités entre documents consultés ainsi que sur les similarités entre profils de consommation d'utilisateurs et d'objets du catalogue. En conséquence, leur utilisation peut contribuer à enclencher une boucle de rétroaction négative où l'utilisateur se voit suggérer une liste de contenus reflétant ses propres biais. À partir de cette liste, l'utilisateur effectue une nouvelle sélection tout aussi biaisée qui complète son profil numérique et qui génère à chaque itération un écosystème informationnel toujours plus retranché sur ses intérêts.

Du côté des études qui mettent l'accent sur les pratiques d'accès à l'information en ligne et sur la notion de chambre d'écho, des sujets d'études en contextes contrôlés ont bien tendance à choisir des articles issus de journaux qui s'alignent avec leurs opinions politiques (Garrett, 2009 ; lyengar et Hahn, 2009 ; Munson et Resnick, 2010). Munson et Resnick (2010) observent toutefois que plusieurs d'entre eux sont aussi à la recherche d'une certaine diversité d'opinions. En outre, il n'est pas prouvé que les consommateurs cherchent à tout prix à éviter la confrontation à des informations contradictoires (Garrett, 2009).

Plusieurs études attestent la présence de phénomènes de fragmentation de l'audience et de regroupements (clusters) sur des blogues ou des microblogues comme Twitter (Adamic et Glance, 2005 ; Barberá et al., 2015; Conover et al., 2011; Garimella et al., 2018), mais également sur la plateforme Facebook (Del Vicario et al., 2017; Jacobson, Myung et Johnson, 2016; Quattrociocchi, Scala et Sunstein, 2016). Cependant, ces études qui sont propres au paysage politique américain sont difficilement généralisables en Europe (Boczkowski et Mitchelstein, 2013 ; Garrett, 2013). De plus, elles ne se limitent qu'à l'étude de thématiques polémiques et de populations très précises. Garimella et al. (2018) relèvent d'ailleurs que toute forme de polarisation disparaît rapidement dès lors que des sujets plus courants sont traités.

Plusieurs auteurs s'accordent sur l'idée que maintenir un réseau social d'une seule couleur politique est une entreprise difficile (Bakshy et al., 2015 ; Dubois et Blank, 2018; Litt et Hargittai, 2016 ; Messing et Westwood, 2014). Cela implique que ces plateformes peuvent également être des espaces d'exposition accidentelle à l'actualité, notamment pour des utilisateurs aux usages moins politisés (Beam et Kosicki, 2014 ; Beam et al., 2017; Fletcher et Nielsen, 2018; Messing et Westwood, 2014 ; Newman et al., 2017 ; Scharkow et al., 2020). 


\section{Arnaud CLAES et al.}

Enfin, plusieurs logiques d'accès à l'information coexistent dans les pratiques médiatiques, que cela soit sur des supports numériques ou non, ce qui contribue à une diversification des sources d'information et des opinions consultées (Dubois et Blank, 2018 ; Flaxman et al., 2016 ; Garrett et al., 2013 ; Newman et al., 2020 ; Yang et al., 2020).

Du côté des études qui s'intéressent aux effets des algorithmes sur différentes plateformes, les résultats sont également mitigés. En ce qui concerne la personnalisation de recherches en ligne, la diversité ne serait que faiblement influencée et le chevauchement des résultats entre différents types d'utilisateurs reste important (Haim, Graefe et Brosius, 2018 ; Krafft, Gamer et Zweig, 2019; Puschmann, 2019). En contrepartie, Claussen, Peukert et Sen (2019) ainsi que Dylko et al. (2017) observent une diminution de la diversité d'articles de presse consultés à la suite de l'usage d'outils de personnalisation. Sur Facebook, Bakshy et al. (2005) observent une baisse de l'exposition à des opinions contraires causée par l'algorithme. Toutefois, le choix des relations sociales des utilisateurs occasionnerait également une baisse de l'exposition dont l'effet serait plus important que celui causé par la personnalisation du flux d'actualités. Aiello et Barbieri (2017) notent enfin une plus grande diversité de consommation par les usagers de Flickr et Tumblr à la suite de l'usage de recommandations automatisées (Aiello et Barbieri, 2017).

Au sujet des études portant sur la recommandation musicale, on observe une diminution de la diversité individuelle (Anderson et al., 2020) couplée, chez Holtz et al. (2020) à une augmentation de la diversité agrégée de l'ensemble des contenus consultés par la communauté d'utilisateurs. Lee et Hosanagar (2019) observent en revanche l'inverse sur un site de vente en ligne. L'étude de Roth, Mazières et Menezes (2020) arrive au constat sur YouTube que le graphe formé par le réseau de recommandations non personnalisées a tendance à confiner l'utilisateur dans un groupement de vidéos homogènes. En se penchant sur le site MovieLens, Nguyen et al. (2014) analysent l'usage d'un système de recommandation et observent qu'il ralentit la diminution de la diversité des contenus consultés observée sur l'ensemble des utilisateurs.

Ces résultats pour le moins disparates soulignent la nécessité d'adopter une perspective sociotechnique plus globale dans l'étude des modalités d'accès à l'information en ligne. L'analyse des usages ne peut se limiter à l'évaluation d'une seule plateforme et doit pouvoir englober un écosystème plus large à même de révéler toutes les nuances et les enjeux propres à ces technologies. 
Algorithmes de recommandation et culture technique : penser le dialogue entre éducation et design

\section{Une perspective écologique pour penser la complexité algorithmique}

L'état de l'art que nous venons d'effectuer confirme la difficulté de construire une théorie générale et souligne les simplifications opérées par des théories comme celles de la bulle de filtres (Bodó et al., 2019). S'il semble actuellement plus ou moins certain que ce que l'on pourrait qualifier de chambre d'écho est un phénomène beaucoup plus poreux qu'il n'y paraît, les effets des algorithmes et leurs appropriations par les utilisateurs reste un mécanisme difficile à discerner. De même que l'utilisateur moyen n'existe pas, un algorithme peut, tout en respectant certains principes généraux, fortement diverger d'une implémentation à une autre.

Cette critique rejoint l'analyse opérée par Bernard Stiegler (2016) pour qui nos objets techniques sont des pharmakons, à la fois remèdes, poisons et bouc-émissaires. Par cette ambivalence, nos techniques, y compris les systèmes de recommandations que nous étudions ici, conservent en elles une potentialité à la fois émancipatrice et aliénante. Comme le souligne Simondon (2012, p. 13) dans l'introduction de son ouvrage Du mode d'existence des objets techniques, "ce qui réside dans les machines, c'est de la réalité humaine ». Dit autrement par Roqueplo (1983, p. 32), nos objets techniques sont « la pétrification des rapports sociaux qu'ils contribuent à la fois à instaurer, à perpétuer et à modifier ». Ce sont, entre autres, ces réalités humaines qui peuvent nous indiquer la voie prise par nos innovations technologiques, que cela soit celle de l'émancipation ou de l'aliénation. Dans le cas qui nous concerne, les stratégies d'hyper-subjectivation poussées par le néolibéralisme (Ouellet, Mondoux et Ménard, 2014) ont contribué à orienter ces technologies de recommandation vers une satisfaction immédiate de besoins à court terme de l'usager, au détriment d'attentes à plus long terme, contribuant à limiter le contrôle de l'usager sur le processus de recommandation (Bodó et al., 2019).

Dépasser une théorie naïve de la bulle de filtres implique donc de plonger dans les conditions concrètes d'existence de ces dispositifs (Roqueplo, 1983) pour saisir les leviers utiles à la mise en œuvre positive de ceux-ci. Nous proposons pour ce faire d'aborder la question sous deux angles complémentaires: (1) l'éducation et (2) le design (qu'il concerne les mécaniques algorithmiques ou les artefacts sémiotiques).

(1) Pour plusieurs penseurs des techniques, l'intégration croissante des technologies au sein de notre environnement 


\section{Arnaud CLAES et al.}

nous a rendus de plus en plus étrangers à notre milieu, qui ne serait plus tant une nature qu'une "technonature " (Roqueplo, 1983). Dépourvu de culture technique (Simondon, 2012), l'individu subit alors une double forme d'aliénation :

D'une part il ne maîtrise pas son propre environnement, d'autre part cette absence de maîtrise le place socialement dans une dépendance permanente par rapport aux organisations et aux individus qui possèdent les compétences qui lui manquent, sans qu'il puisse contrôler la manière dont ils exercent ces compétences et interviennent ainsi, à travers son environnement, sur sa propre existence. (Roqueplo, 1983, p. 37)

Cette culture technique est alors à comprendre comme un ensemble de savoirs et savoir-faire permettant de penser la genèse de la technique et ses modalités d'intégration et de relation à un contexte spécifique. Elle doit permettre de se réapproprier cet environnement qui nous est devenu de plus en plus étranger (Chouteau et al., 2015; De Noblet, 1981; Roqueplo, 1983). Cette conception de la culture technique complète ici le projet de développement d'une littératie médiatique des individus comme condition d'émancipation (Roqueplo, 1983 ; Fastrez et Philippette, 2017).

Parce que « les ingénieurs ne fabriquent pas uniquement un outil mais la société même dans laquelle nous vivons » (Roqueplo, 1983, p. 36), cette perspective complète les préoccupations traditionnelles de l'éducation aux médias en soulignant le rôle de la culture comme opérateur social. Pour ces raisons, afin de cerner ces « connaissances fonctionnellement pertinentes ॥ (Roqueplo, 1983, p. 205), le premier cas d'étude que nous aborderons dans les pages suivantes s'intéressera aux représentations et attitudes que des jeunes utilisateurs développent au contact de ces dispositifs et qui conditionnent leurs comportements d'adaptation aux phénomènes de captation de données et de subjectivisation.

(2) Se limiter à cette perspective impliquerait que nous plaçons la responsabilité critique du seul côté de l'usager. Pourtant, l'absence de culture technique découle également d'un développement technologique et économique qui empêche toute forme de critique à travers une pratique du design qui tente de lisser un maximum le conflit et l'altérité pour apparaître comme immédiate (Hamilton et al., 2014). Propulsées par leur popularité et leurs positions de quasi-monopoles, les plateformes numériques principales contribuent aujourd'hui à l'infrastructure de nos sociétés modernes, au même titre que nos réseaux électriques ou ferroviaires (Plantin et al., 2018). En obtenant au sein de l'espace social ce statut d'infrastructure, ces dispositifs 
Algorithmes de recommandation et culture technique : penser le dialogue entre éducation et design

disparaissent de l'horizon politique, reléguant ainsi leur gestion aux techniciens qualifiés et invisibilisant progressivement leurs rouages internes aux yeux des utilisateurs (Star et Ruhleder, 1996).

Ce second constat nous a amenés à la réalisation du second cas d'étude ci-dessous, dans le but d'interroger la façon dont des technologies de recommandation peuvent visibiliser les opérations de filtrage et incarner davantage une solution à la manière d'un partenaire d'accompagnement de la recherche de contenus plutôt qu'à celle d'un assistant tentant de prédire les besoins.

Si cet article n'a pas la prétention de concevoir une " thérapeutique " de nos systèmes informatiques (Stiegler, 2016), nous proposons ici de discuter d'une écologie de l'esprit, comprise comme l'observation des relations constitutives de notre milieu technique et des pistes de rétablissement d'un équilibre malmené (Bateson, 2008; Charbonnier et Kreplak, 2012 ; Guattari, 2005). De la sorte, nous espérons envisager un progrès alternatif : plutôt que de rendre ces outils toujours plus " intelligents ", la question est de savoir comment vivre en bonne intelligence avec eux sans pour autant verser dans une perspective utilitaire?

\section{3 Étude de cas 1 : analyse des représentations des bulles de filtres chez les jeunes}

\subsection{Protocole méthodologique: entretiens semi- directifs}

Cette section rend compte des résultats de l'analyse de vingtsept entretiens semi-directifs ${ }^{1}$ réalisés entre avril et juin 2019 auprès de jeunes Bruxellois âgés de 15 à 25 ans, mais aussi d'expertes et experts de l'éducation aux médias ainsi que d'enseignants et enseignantes. À travers ce dispositif, il s'agit d'identifier directement les représentations potentielles qu'ont les jeunes de l'existence de bulles de filtres et de l'influence des algorithmes de recommandation. L'analyse est indirectement complétée par les discours d'acteurs en contact récurrent avec les jeunes. L'objectif est aussi de rendre compte des tactiques (de Certeau, 1990) développées par les jeunes vis-à-vis des

\footnotetext{
1 Les entretiens sont issus d'un dispositif méthodologique plus large comprenant dix-neuf focus groups semi-directifs et la collecte de données quantitatives dans le cadre du projet de recherche Alg-Opinion financé par l'organisation régionale bruxelloise pour la recherche et l'innovation (Innoviris) et le programme Anticipate [2018-ANTICIPATE-24].
} 


\section{Arnaud CLAES et al.}

systèmes de recommandation. À l'image des entretiens menés auprès de jeunes américains visant à saisir leur conscience des filtres sur les réseaux sociaux (Powers, 2017) ou de l'analyse de tweets relatifs à l'expérience d'internautes sur les filtres existants sur Facebook (Bucher, 2016), notre approche qualitative permet d'accéder à la "boîte noire " (Kitchin, 2016) des effets des algorithmes sur la diversité des informations au travers des discours des jeunes.

Concrètement, les entretiens ont été réalisés à l'aide d'un guide comprenant trois thèmes généraux ${ }^{2}$ dont les questions ne définissent pas directement l'objet des rencontres afin de voir si la question des systèmes de recommandation et des bulles de filtres émergent spontanément du terrain. Dans une optique proche de la théorie ancrée (Keller, 2013 ; Strauss et Corbin, 1994) qui a pour objectif de faire émerger des catégories et concepts des données elles-mêmes, nous avons réalisé une analyse thématique inductive en "catégories conceptualisantes " (Paillé et Mucchielli, 2016). Nous avons ainsi lié analyse du corpus et conceptualisation des données en associant certains extraits des transcriptions à des catégories qui les décrivent, et nous avons évolué ensuite par addition d'extraits supplémentaires. Après un retour d'expériences entre chercheurs, il a été décidé de se concentrer sur les extraits où les jeunes font référence à des mécanismes révélant l'influence des algorithmes de recommandation et des interfaces des réseaux sociaux, avec un focus sur les discours relatifs à l'identification d'une bulle de filtres. L'objectif est de saisir les mécanismes au travers desquels les jeunes prennent conscience de l'existence de systèmes de recommandation. Sur la base de ces extraits, les analyses des entretiens ont permis d'identifier des discours relatifs (1) aux mécanismes qui rendent visibles les systèmes de recommandation, (2) à la perception de ces filtres, et (3) aux tactiques développées pour les moduler.

\subsection{Premiers résultats qualitatifs}

De manière générale, comme mis en évidence par de précédentes recherches (par exemple Heaton, Millette et Proulx, 2012), les trois catégories d'individus interrogés laissent entendre que les jeunes ont « quitté » Facebook pour contourner la présence des adultes et éviter un certain " contrôle social ». Ils utilisent massivement Instagram pour la communication dite de " un-vers-plusieurs » au travers du partage de photos ou de

\footnotetext{
2 Les trois thèmes sont (a) l'usage des plateformes et la consommation médiatique (b) les perceptions du fonctionnement des plateformes et (c) les représentations de l'actualité.
} 
Algorithmes de recommandation et culture technique : penser le dialogue entre éducation et design

stories accompagnées de textes et YouTube pour la consommation de vidéos. Ils choisissent également des plateformes de type Messenger, Snapchat ou WhatsApp pour la communication de "pair-à-pair ». Quelques-uns naviguent sur des plateformes jugées encore de niche en fonction de leurs intérêts comme Twitter ou Twitch pour le sport, la musique ou les jeux vidéo. Les individus interrogés constatent aussi que les dispositifs sociotechniques ne fonctionnent pas indépendamment les uns des autres (tweets sur Instagram, liens YouTube sur Facebook, article web sur WhatsApp, etc.), les jeunes relevant d'ailleurs de plus en plus de mimétisme entre les plateformes. Ces observations sont globalement comparables aux résultats de recherches menées parmi des jeunes occidentaux, comme aux États-Unis (Anderson et Jiang, 2018). Sur la base de ces constats, notre analyse se concentre sur les discours des jeunes, des enseignants et des experts à propos des plateformes Instagram et YouTube.

\subsubsection{Les mécanismes révélant l'existence de filtres}

La plupart des enseignants dénoncent l'influence des systèmes sociotechniques sur l'émergence de certains comportements considérés comme problématiques chez les jeunes, comme le cyberharcèlement (Enseignant 1), la radicalisation (Enseignant 5), le partage des théories conspirationnistes (Enseignant 2) ou de fausses nouvelles (Enseignant 4). Ces enseignants n'associent pas ces problématiques à la question des algorithmes et des bulles de filtres, au contraire des experts. Ces derniers précisent que ces comportements restent cependant assez marginaux et que d'autres publics peuvent également être concernés. Ils estiment d'ailleurs que les jeunes sont capables d'identifier les phénomènes de filtrage et donc indirectement l'existence des algorithmes, même si ces derniers n'utilisent pas toujours ces termes pour verbaliser leur pensée.

Ainsi, lors des entretiens avec les jeunes, on remarque que certains font référence à différents mécanismes qui rendent ces filtres visibles. En ce sens, un jeune note la préférence donnée par Instagram aux publications de certains de ses amis :

Quand un ami publie quelque chose, ça arrive directement en premier dans mes trucs. Mais pas tout le temps, parfois ça ne le met pas et du coup, je tombe dessus par pur hasard. (Jeune 8) 


\section{Arnaud CLAES et al.}

Un autre (Jeune 6) confirme que la plupart des plateformes utilisées organisent son " fil », et donc ce qu'il consulte lors de chaque connexion en fonction des "gens " qu'il suit ou des actions qu'il entreprend.

À l'inverse, certains jeunes n'ont pas de conscience des mécanismes de filtrage et de recommandation qui opèrent. Avec beaucoup d'hésitations, certains remarquent plutôt que les réseaux leur permettent d'accéder à autant d'informations que la télévision :

Je trouve que c'est un peu la même chose sauf que, comme j'ai dit, à la télé ils nous mettent un peu ce qu'ils veulent. Et dans les réseaux, ben il y a tout. (Jeune 2)

$\mathrm{Du}$ fait du relatif secret qui entoure la conception des algorithmes, les représentations qu'en ont des jeunes sont parfois exagérées, fantasmées ou sont fondées sur des croyances non vérifiées et non sur des expériences concrètes prises en exemple pour appuyer leurs propos:

Comme je l'ai dit tout à l'heure, j'ai l'impression parfois qu'on est suivi. Donc franchement tout ce que je fais, après il y aura des publicités, des vidéos, etc. Tout est relié j'ai l'impression. Parfois, j'ai l'impression que lorsque je regarde quelqu'un sur Instagram, après sur YouTube on me le propose. On dirait qu'ils se sont passé le mot. Tout ce que je fais semble relié. C'est un petit peu bizarre, ça me perturbe personnellement parce que j'ai vraiment l'impression que tout est suivi. (Jeune 7)

Même si la plupart des jeunes sont capables d'identifier l'existence de filtres en notant que rien n'est le fruit du "hasard", ils reconnaissent parallèlement leur incompréhension des mécanismes qui opèrent en profondeur. Leurs hésitations rendent compte de la boîte noire que constituent les systèmes de filtres :

Je ne peux pas vraiment dire que c'est du hasard parce que j'ai compris un peu le système. Quand on clique sur une vidéo, ça va analyser à peu près toute la communauté de cette personne. Qui regarde quoi, etc.? Et après ça va nous recommander ce genre de vidéos. (Jeune 8 )

D'autres mécanismes permettent aux jeunes de percevoir l'existence de filtres. Ainsi, la comparaison entre son profil et celui d'autres utilisateurs permet à une jeune (Jeune 9) d'identifier différents systèmes de recommandation: elle 
Algorithmes de recommandation et culture technique : penser le dialogue entre éducation et design

explique que son copain ne voit que des photos de football sur « Insta » alors qu'elle voit principalement des photos de «fringues". En outre, elle se sent capable d'accéder à une diversité d'informations si elle lance des recherches actives sur la plateforme. Comme nous le verrons dans la suite de nos analyses, rechercher activement des contenus sur d'autres plateformes est l'une des tactiques adoptées par des jeunes pour atténuer la bulle à laquelle ils se sentent confrontés.

\subsubsection{Des filtres contestés, appréciés ou invisibles}

Les jeunes interrogés évaluent différemment les effets induits par les algorithmes sur leur accès à une diversité d'informations. Certains dénoncent explicitement l'existence de «bulles » et la nécessité d'en sortir.

Je n'aime pas trop être enfermé dans une bulle, si c'est le cas, on ne sait plus trop ce qui se passe à l'extérieur et dès qu'on sort de cette bulle on est perdu. Je trouve donc important de se tenir informé de ce qui se passe autour, plus que notre propre centre d'intérêt. (Jeune 3 )

Si je ne recherche pas vraiment, je ne pense pas que j'aurai vraiment accès à ça [ndr: un sujet particulier]. Mais j'ai l'impression que maintenant les réseaux ne nous proposent que ce à quoi on est abonné ou quoi alors que parfois on a envie de découvrir. C'est vraiment que si je parle avec quelqu'un et qu'il m'en parle [ndr : d'un sujet], que je dois aller rechercher... Et puis ça va arriver dans mon fil. (Jeune 7)

Cette observation est également partagée par les enseignants qui notent que les technologies utilisées par les jeunes ne leur " ouvrent pas le monde » (Enseignant 6); ils lient ce problème au manque d'informations et d'éducation sur le sujet (Enseignant 3).

Des jeunes estiment cependant qu'il est normal qu'il existe une personnalisation des contenus par un système de recommandation. Certains affirment en outre que ces algorithmes influencent relativement peu ce qui leur est proposé, car il suffit de s'intéresser à quelque chose de nouveau pour qu'il y soit intégré. Quelques-uns considèrent même que les dispositifs sociotechniques ne créent pas de "bulles " et qu'ils permettent au contraire de s'en détacher. Et d'autres, enfin, s'ils identifient un phénomène de recommandations, ils avancent que cela les aide à découvrir des contenus habituellement peu 
Arnaud CLAES et al.

accessibles. Les jeunes cadrent donc cette problématique des filtres de manière assez contrastée.

2.2.3. Des tactiques aidant à influencer les effets des algorithmes

Certains jeunes développent des stratégies ou plutôt des " tactiques " (de Certeau, 1990) pour contourner les effets des algorithmes sur leurs écosystèmes médiatiques. Celles-ci ne sont pas uniquement utilisées pour lutter contre les phénomènes de bulles de filtres ; certains jeunes tentent au contraire de les accentuer. Comme nous l'avons vu précédemment, tous les jeunes ne considèrent pas comme étant problématique l'accès à des informations ajustées en fonction de leurs préférences. Les filtres ne leur posent pas de "problème " en soi. Bien au contraire, certains relèvent leur utilité : les filtres leur évitent d'être parasités par ce qui ne les intéresse pas. Certains commentaires révèlent aussi que des jeunes n'ont pas pleinement conscience des contenus accessibles.

Quand il s'agit d'influencer directement l'accès aux informations et les filtres existants sur le réseau, des jeunes expliquent leurs tentatives pour contrôler activement les plateformes sur lesquelles ils sont connectés. Pour certains, il s'agit de s'abonner à des chaînes YouTube spécifiques ou de suivre des comptes sur Instagram pour influencer les algorithmes.

Non, sur YouTube ça a pas l'air de me déranger [ndr : que les contenus soient triés], mais sur Instagram avant ça me dérangeait pas forcément, mais maintenant ça me dérange. C'est pour ça que je me suis abonnée à beaucoup de comptes. (...) Parce que du coup j'avais l'impression de voir tout le temps la même chose, et ça me posait problème même au niveau de l'actualité parce que j'avais l'impression que j'étais... Allez, c'est bien d'être informé par les autres mais j'aimerais bien aussi des suggestions qui apparaissent des médias, par exemple avoir CNN ou un autre média. (Jeune 1)

À l'inverse, d'autres jeunes expliquent tenter d'influencer l'information reçue en se désabonnant de comptes. Ces tactiques d'abonnements-désabonnements qui visent l'évaluation des mécanismes d'ajustement des algorithmes par ces jeunes semblent également participer à une meilleure prise de conscience des effets multiples d'un comportement plus actif de leur part sur leur environnement informationnel. En effet, 
Algorithmes de recommandation et culture technique : penser le dialogue entre éducation et design

comme le relèvent certains d'entre eux, l'accès à des recommandations issues d'une multitude de comptes peut renforcer la diversité de l'information, mais cela favorise également le zapping, la lecture des titres et non des contenus ou encore le survol plutôt que le positionnement. Les tactiques de certains jeunes ne visent donc pas à diversifier les contenus reçus mais bien à les cibler davantage.

Une autre tactique consiste à fournir activement à l'algorithme des informations personnelles à travers des actions spécifiques. L'extrait suivant met en évidence par exemple comment un jeune influence activement les tris des algorithmes en évitant de liker certains contenus :

Mmmmmmh... Ben des fois par exemple quand quelque chose m'intéresse pas, je fais ça (note: montrant sur le téléphone en direct). Je clique par exemple, « ceci ne m'intéresse pas ". Je fais ça et je vais genre voir moins de posts comme ça. (...) Oui, parce que j'ai remarqué que quand je likais quelque chose, ou quand j'envoyais ça à mes potes, ben j'avais de plus en plus de posts de ce genre. Du coup, des fois par exemple, si je voulais pas avoir, je sais pas, des chanteurs dans mon fil d'actualité et bien j'arrêtais de liker. Et du coup voilà. En plus c'est vraiment varié du coup j'avance et je regarde un peu ce qui m'intéresse. (Jeune 2)

Enfin, les jeunes ont également recours à des tactiques plus classiques en vue de diversifier les informations auxquelles ils sont confrontés, en effectuant des recherches actives via différentes plateformes (et conséquemment via plusieurs systèmes de recommandation) ou simplement en discutant avec des amis, les enseignants ou leur famille. Comme on l'observe dans les discours des jeunes, l'information reçue sur les réseaux sociaux est comparée plus ou moins activement aux actualités diffusées à la télévision généralement regardée par leurs parents. L'ensemble de ces éléments rappellent l'importance de considérer l'usage des réseaux dans un contexte informationnel plus large.

En résumé de cette première étude de cas, nous pouvons souligner que la problématique de la bulle de filtres ne peut être traitée de manière simple et univoque, au risque de promouvoir des solutions censées répondre à l'invisibilisation des systèmes de recommandation par l'introduction "forcée » d'une plus grande diversité de contenus. Or le constat à la fois d'une problématique vécue différemment par les jeunes et de tactiques adoptées pour certains afin de renforcer le phénomène invite à considérer que la solution au problème, s'il en est, passe par une 
éducation tenant davantage compte de ces réalités d'usages en vue de développer une culture numérique et une littératie médiatique émancipatrice. Ce constat ne retire cependant en rien la nécessité de penser une technique qui permette un accès à la diversité et de faire des choix plus éclairés. C'est ce que nous abordons dans notre deuxième étude de cas.

\section{4 Étude de cas 2: deux solutions pour une algorithmisation partenaire de la consommation de contenus}

\subsection{Protocole méthodologique}

Cette deuxième étude de cas aborde la problématique de la bulle de filtres sous l'angle de l'ingénierie mathématique, à partir de la synthèse de deux recherches dirigées au sein de l'École Polytechnique de Louvain (Laurinaviciute, 2019; Mottet et Moumal, 2019). Ces deux recherches ont été menées à partir d'un large jeu de données (anonymes) d'un mois de consommation d'une plateforme de contenus audiovisuels (RTBF Auvio ${ }^{3}$ ). Si ces recherches se sont orientées vers des modélisations et tests de natures différentes, elles ont toutes deux l'objectif initial d'évaluer les facteurs d'influence des bulles de filtres.

Avant d'entrer dans les résultats de ces études, il convient de souligner qu'une analyse fonctionnelle d'algorithmes de recommandation exposés aux individus s'avère être une tâche complexe. Tout d'abord, les algorithmes de recommandation ont pour vocation de maintenir l'attention de leur public cible, et leurs détails d'implémentation sont donc rarement partagés par les responsables des plateformes qui les utilisent. En effet, pour la majorité des acteurs du secteur, l'attention représente une source importante de revenus (Citton, 2014). Évaluer leurs effets sur le long terme reste dès lors en partie hypothétique, puisqu'il faut simuler "hors ligne » (offline approach) leurs comportements sur des jeux de données réelles. Par ailleurs, ces algorithmes s'appuient de plus en plus sur l'analyse de quantités importantes de données sans modèle sous-jacent. Cette approche basée sur des mécanismes d'auto-apprentissage a prouvé sa pertinence dans de nombreuses applications (Bobadilla, Ortega, Hernando et Gutiérrez, 2013), mais a pour défaut majeur de ne pas identifier de relations évidentes et naturelles entre les données disponibles et, à l'inverse, d'établir

\footnotetext{
3 Plateforme de contenus en ligne de la Radio-Télévision Belge Francophone de service public.
} 
Algorithmes de recommandation et culture technique : penser le dialogue entre éducation et design

des résultats sur la base de convolutions profondes (Sinha et Swearingen, 2002). II en découle que les plateformes disposant des plus grandes quantités de données obtiennent un avantage considérable dans la création de leur algorithme de recommandation et que leur régulation effective dans le temps ne s'en trouve que plus complexe.

Afin de pouvoir être conceptualisée mathématiquement, une bulle de filtres peut être définie en recourant au concept de communauté dans une structure mathématique appelée graphe. Un graphe permet de représenter la similarité entre différents utilisateurs afin d'en extraire une organisation hiérarchique. Spécifiquement, deux utilisateurs ont une similarité élevée s'ils consomment une proportion importante de contenus identiques. Il a été observé que, naturellement, de tels réseaux développent des structures de communauté (Browet, 2014 ; Leskovec et al., 2010), c'est-à-dire qu'ils se composent de groupes denses d'utilisateurs fortement similaires entre eux et dissimilaires à tous les autres. Dans le cas qui nous occupe, une manière naturelle de représenter les similarités entre contenus est de construire un graphe dont chaque nœud représente un contenu, et dont deux contenus sont reliés par une arête si un grand nombre d'utilisateurs a consommé les deux contenus. On obtient ainsi une représentation combinatoire de l'interrelation entre différents contenus.

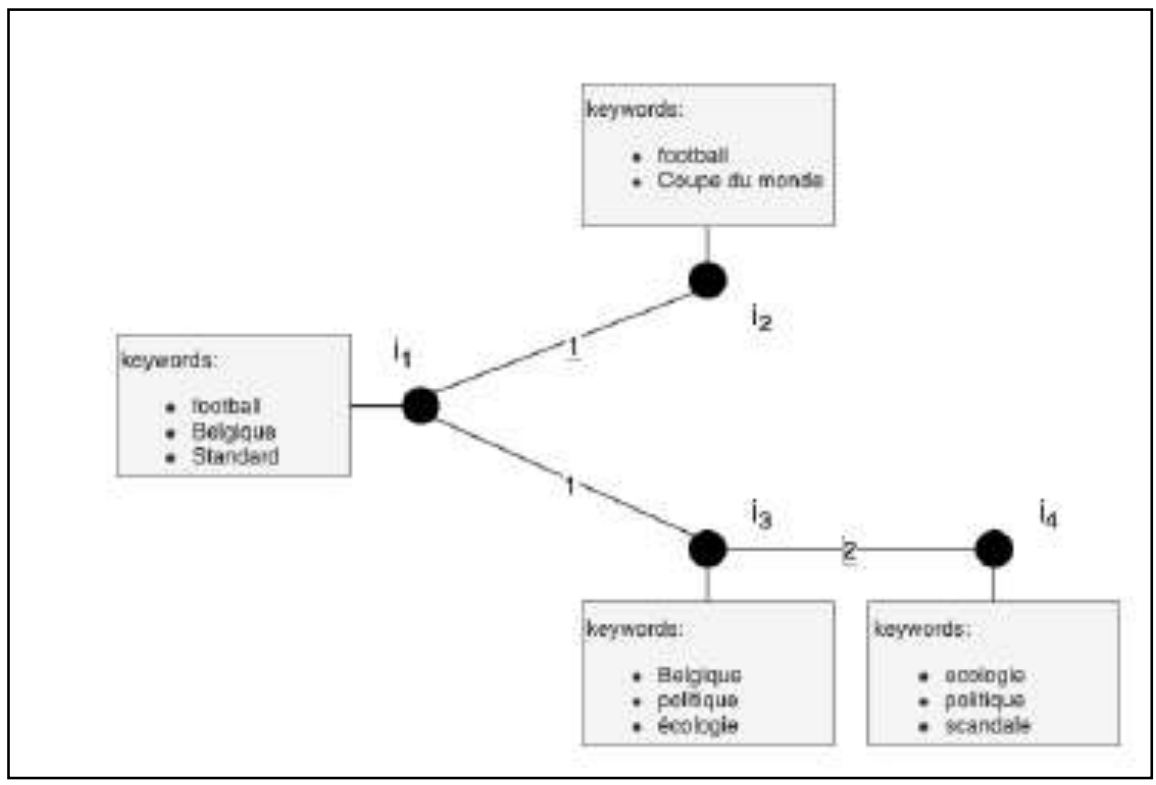

Figure 1. Exemple de graphe basé sur les mots-clés des contenus (Mottet et Moumal, 2019, p. 50) 


\section{Arnaud CLAES et al.}

Un phénomène de bulle de filtres apparaît à partir du moment où (1) les recommandations proposées à un utilisateur ont une probabilité importante d'appartenir aux centres d'intérêts dominants de sa/ses communautés (phénomène de rétroaction positive), et (2) un contenu divergent des centres d'intérêts d'une communauté met un temps important à percoler au travers des recommandations (faible probabilité de consommation). Afin d'en étudier l'émergence et de proposer une solution à ce phénomène, la première recherche (Mottet et Moumal, 2019) est partie du jeu de données de la plateforme RTBF Auvio pour simuler en laboratoire les effets d'algorithmes traditionnels (par exemple classic content-based, k-nearest neighbors, matrix factorization, singular value decomposition) sur les recommandations de contenus, et évaluer ces résultats au regard de métriques servant généralement à évaluer la qualité de ces recommandations (par exemple accuracy, coverage, diversity, serendipity et robustness), pour ensuite proposer une solution informatique au phénomène étudié. La seconde recherche (Laurinaviciute, 2019), quant à elle, consistait d'abord en une étude plus théorique des systèmes de filtres en science des données (par exemple collaborative filtering, content-based filtering, demographic filtering, knowledge-based filtering) pour ensuite se tourner vers le design d'une solution algorithmique avancée afin de faire sortir l'utilisateur de sa bulle hypothétique. Pour les détails techniques des protocoles d'analyse de ces deux recherches, nous renvoyons un lectorat aguerri vers ces travaux.

\subsection{Deux propositions de conception}

\subsubsection{B-score, recommandation hybride et interface de choix d'algorithme}

Afin d'évaluer la performance des différents algorithmes sur l'ensemble (set) de données collectées auprès de la plateforme RTBF Auvio, les auteurs ont dû préalablement entraîner les algorithmes en créant d'un côté un jeu d'entraînement (training set) et de l'autre un jeu sur lequel porte l'évaluation (test set). Cet entraînement est en effet nécessaire afin d'éviter les biais d'interprétation du comportement d'un algorithme qui ne se serait jamais ajusté à un jeu de données (problème appelé cold start dans le jargon de l'ingénierie). Préalablement à cela, il convenait de nettoyer l'ensemble des données ${ }^{4}$. Sur les 294000 items que comprenait la base de données RTBF Auvio, 187271 étaient accessibles aux utilisateurs sur la plateforme au moment de la

\footnotetext{
4 Le fichier log en lui-même reprenant l'historique des actions des utilisateurs faisant environ 45 Go en version compressée, et dix fois plus après décompression.
} 
Algorithmes de recommandation et culture technique : penser le dialogue entre éducation et design

recherche (Mottet et Moumal, 2019, p. 24). Au rythme actuel, environ 300 nouveaux contenus sont publiés chaque jour sur cette plateforme. Ces contenus couvrent 3979 programmes distincts, classifiés en 113 catégories, que les auteurs de la première recherche ont ramené à 6 catégories élémentaires (divertissement, jeunesse, séries, info, vie pratique et sport). Ce travail de préparation des données permet, pour chaque contenu, d'identifier les catégories qui lui sont associées, ainsi que les mots-clés par ailleurs extraits en vue d'établir un graphe hiérarchique (cf. supra).

Dans leur recherche, Mottet et Moumal se sont ensuite intéressés à une donnée particulière extraite du fichier de logs : le croisement entre des utilisateurs (identifiant unique codé en 32 bits) et leur consommation à plus de $90 \%$ d'un contenu vidéo donné (Mottet et Moumal, 2019). De la sorte, ils ont pu établir parallèlement un historique de consommation pour chaque utilisateur, et créer des profils de consommation au regard des catégories de contenus. Leur objectif étant de proposer un système de recommandation "idéal ", ils ont dans un premier temps évalué les effets de chacun des algorithmes traditionnels (cf. supra) sur les données au regard de mesures de précision (accuracy ou R-score). Ensuite, ils ont voulu évaluer le risque de chacun de ces algorithmes d'enfermer un utilisateur dans une bulle de contenus (user bubbleness). Pour pouvoir faire cela, ils sont partis d'une méthode d'analyse par clusters (ou " communautés ", cf. supra), sur la base d'un côté des contenus (item-based) et de l'autre des utilisateurs (profiles). Ils ont ensuite introduit une mesure, le $B$-score, afin d'évaluer combien une liste de recommandations pourrait influencer les différents profils d'utilisateurs à " sortir de leur bulle ». Au-delà d'une évaluation de la mesure classique de diversité (diversity), ce B-score compare le système de recommandation avec l'historique de consommation de chaque profil d'utilisateur (Mottet et Moumal, 2019 , p. 64). Le constat que leur recherche pose à travers diverses analyses est qu'il n'y a pas d'optimum absolu au niveau des solutions algorithmiques traditionnelles, une augmentation de la précision se faisant généralement au détriment de la diversité et vice versa.

Partant de ce constat, les auteurs proposent une double solution innovante. Le premier volet de cette solution consiste à fusionner les listes de recommandations produites par ces différents algorithmes de sorte à fournir une recommandation hybride, combinée avec une re-pondération de certains contenus selon qu'ils appartiennent ou non aux clusters habituels de consommation de l'utilisateur (Mottet et Moumal, 2019, p. 71). Le 


\section{Arnaud CLAES et al.}

deuxième volet, quant à lui, consiste à développer une solution concrète, en utilisant un calcul de points frontières dans les recommandations (frontière de Pareto) à l'aide de techniques d'optimisation (pour les détails, voir Mottet et Moumal, 2019, p. 78 et suiv.), permettant à l'utilisateur, à travers une interface graphique, d'influencer les recommandations qui lui sont faites en déterminant ses préférences en termes de « précision » et de " diversité », et conséquemment les paramètres de l'algorithme traitant les données.

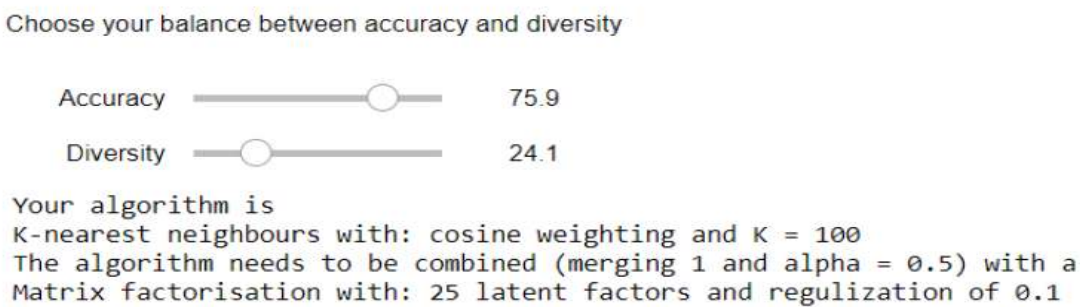
p. 78)

Figure 2. Illustration de la solution appelée trade-off slider (Mottet et Moumal, 2019,

\subsubsection{Agir sur les bulles les plus résistantes}

L'auteure de ce deuxième travail propose quant à elle une solution algorithmique en vue de sortir les utilisateurs de leurs zones de confort de consommation. Elle propose en effet une notion quantifiée de bulle de filtres sur la base d'un principe de « distance affinitaire » des utilisateurs vis-à-vis de certains contenus (Laurinaviciute, 2019, p. 22 et suiv.). Autrement dit, il y a « bulle de filtres » dès lors qu'une personne ne consomme que majoritairement des contenus proches de son profil initial. Elle conceptualise ainsi la bulle de filtres comme plus ou moins résistante à l'influence de facteurs externes, en l'occurrence des informations non congruentes. Pour sortir l'utilisateur de cette tendance, sur la base de principes de conductance et de résistance inspirés des mathématiques appliquées aux réseaux électriques (Laurinaviciute, 2019, p. 46), elle propose une solution algorithmique de recommandation (« crossing the river algorithm ») visant à suggérer successivement à un utilisateur " enfermé " des contenus suffisamment proches de ses habitudes pour l'intéresser, mais suffisamment éloignés pour l'inviter à sortir de sa zone de consommation habituelle. Le principe étant alors de mathématiquement générer un chemin idéal de diversification, plus ou moins long selon la "résistance ", entre contenus consommés et contenus suggérés (« conductance »). 
Algorithmes de recommandation et culture technique : penser le dialogue entre éducation et design

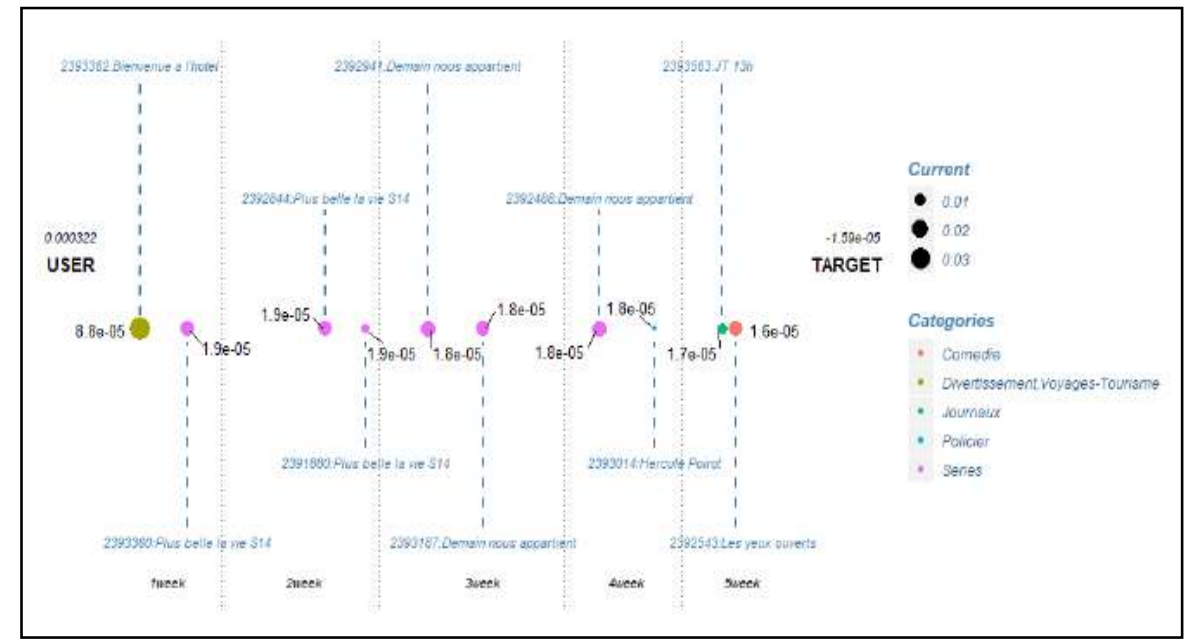

Figure 3. Illustration de l'algorithme crossing the river: 10 contenus ayant une affinité mutuellement proche sont successivement recommandés à un utilisateur afin de lui faire découvrir la catégorie Téléfilm (dans Laurinaviciute, 2019, p. 50)

Ces premières solutions permettent de repenser la problématique de la bulle de filtres et l'accompagnement technique de l'utilisateur dans sa consommation informationnelle. Elles restent bien évidemment exploratoires et posent encore de nombreuses questions liées à leur implémentation comme la détermination du contenu cible dans le cas ci-dessus. Elles ouvrent cependant la voie à des environnements techniques davantage soucieux des besoins des utilisateurs et cherchant à équilibrer diversité et pertinence des informations.

\section{Conclusion}

L'étude des pratiques informationnelles de jeunes a permis de mettre en évidence la disparité des appropriations de ces dispositifs algorithmiques. Les représentations construites par l'utilisateur sur la base de savoirs parcellaires conduisent à l'élaboration de tactiques en phase avec leur attitude à l'égard du processus de filtrage. Plus l'usager peut accumuler des indices sur le mécanisme interne à la boîte noire, plus il semble développer des comportements visant à reprendre le contrôle sur le dispositif. En ce sens, la composition d'un savoir technique fonctionnellement utile contribue à diminuer l'aliénation de l'usager. Même lorsque ces représentations ne sont pas parfaitement en concordance avec le mécanisme réel, le principe de questionnement incite l'utilisateur à diversifier ses pratiques informationnelles pour accéder à d'autres sources d'informations mobilisant d'autres logiques structurelles (presse " classique ", recherche en ligne ou sur d'autres réseaux sociaux, etc.). Cette 
attitude ouvre la porte au développement de dispositifs qui pourraient tirer profit de l'engagement de l'utilisateur en l'invitant à agir concrètement sur certains fonctionnements internes. Cela ne peut arriver qu'à condition de donner les «prises" (Chateauraynaud, 2006) nécessaires à l'usager pour comprendre sa consommation et le comportement du dispositif en fonction de ses intentions.

Le travail de conception, réalisé dans un deuxième temps par des étudiants en sciences des données et en sciences informatiques, nous a permis de questionner les modélisations communément admises dans le domaine des systèmes de recommandation. Les dispositifs proposés n'évacuent pas totalement la logique initiale du système de recommandation, qui continue à se baser sur des données d'usagers de sorte à leur fournir une série de recommandations automatisées. Néanmoins, la modélisation des utilisateurs et de leurs intérêts à l'aide d'outils issus de la théorie des graphes nous a permis de démontrer la possibilité de développer des algorithmes de recommandation qui s'adaptent aux consommations de l'utilisateur par des mécanismes d'apprentissage et permettent de le guider vers des contenus d'un certain type pré-spécifié dans une approche incrémentale et progressive. Ces algorithmes ont la particularité de réagir à l'apparition, dans l'historique de navigation de l'utilisateur, de clusters de contenus trop concentrés indiquant une potentielle « chambre d'écho » ou «bulle de filtres ».

Ces approches se distinguent de dispositifs plus classiques, en reconnaissant l'indécidabilité d'une stratégie optimale de recommandation. L'impossibilité de maximiser en même temps la diversité et la précision n'est pas ici considérée comme une faiblesse du système, mais plutôt comme sa force. Plutôt que d'imposer un régime particulier, généralement la satisfaction de mesures de précisions, le choix opéré ici consiste à laisser l'orientation globale à un acteur humain. II est alors possible soit de fixer le poids accordé à l'une ou l'autre métrique, soit de fixer une direction vers laquelle on souhaite diversifier le profil de consommation de l'utilisateur. Comme nous l'avons déjà signalé plus tôt, ces technologies, dont les deux prototypes discutés ici, ne peuvent pas assurer à elles seules le développement d'opinions mieux informées. Néanmoins, en permettant à l'utilisateur d'opérer une certaine forme de contrôle, ces outils permettent une revisibilisation des processus techniques qui, pour certains profils d'utilisateurs, seraient autrement indétectables. 
Algorithmes de recommandation et culture technique : penser le dialogue entre éducation et design

En juxtaposant ces deux approches, nous avons voulu souligner leur nécessaire réciprocité. En constatant la faisabilité technique d'une recommandation plus "transparente », nous éclairons d'autant plus l'importance d'une forme « d'acculturation technique ". Cette dernière se doit d'être promue tant près des ingénieurs responsables de leur implémentation dans notre espace public qu'auprès des usagers qui doivent pouvoir saisir les opportunités d'action qui leur sont proposées. Simultanément, en recherchant des processus de conception alternatifs, nous essayons de promouvoir un changement technique comme espace d'affirmation plutôt que de négation sociale (Roqueplo, 1983). C'est également en pensant les limites de ces dispositifs que nous pouvons définir là où l'humain peut reprendre l'initiative. Loin de libérer l'usager de toute forme de responsabilité, la délégation de fonctions cognitives promue par ces technologies demande qu'il s'investisse plus que jamais dans les contrats de lecture qu'il négocie avec elles.

\section{Références bibliographiques}

Adamic, L. A. et Glance, N. (2005, août). The political blogosphere and the 2004 U.S. election: Divided they blog. Communication présentée au $3^{\text {rd }}$ International Workshop on Link Discovery, Chicago (États-Unis). doi : https://doi.org/10.1145/1134271.1134277

Aiello, L. M. et Barbieri, N. (2017, février). Evolution of Egonetworks in Social Media with Link Recommendations. Communication présentée à la Tenth ACM International Conference on Web Search and Data Mining, Cambridge (Royaume-Uni). doi : https://doi.org/10.1145/3018661.3018733

Anderson, A., Maystre, L., Anderson, I., Mehrotra, R. et Lalmas, M. (2020, avril). Algorithmic Effects on the Diversity of Consumption on Spotify. Communication présentée à la Web Conference 202 0, Tapei (Taïwan). doi : https://doi.org/10.1145/3366423.3380281

Anderson, M. et Jiang, J. (2018). Teens, Social Media \& Technology 2018 [Rapport du Pew Research Center (Internet \& Technology)]. Repéré à :

https://www.pewresearch.org/internet/2018/05/31/teens-socialmedia-technology-2018/. 


\section{Arnaud CLAES et al.}

Bakshy, E., Messing, S. et Adamic, L. A. (2015). Exposure to ideologically diverse news and opinion on Facebook. Science, 348(6239), 1130-1132.

doi : https://doi.org/10.1126/science.aaa1160

Barberá, P., Jost, J. T., Nagler, J., Tucker, J. A. et Bonneau, R. (2015). Tweeting From Left to Right: Is Online Political Communication More Than an Echo Chamber? Psychological Science, 26(10), 1531-1542.

doi : https://doi.org/10.1177/0956797615594620

Bateson, G. (2008). Vers une écologie de l'esprit (Tome 2). Paris, France : Éditions du Seuil.

Beam, M. A. et Kosicki, G. M. (2014). Personalized News Portals: Filtering Systems and Increased News Exposure. Journalism \& Mass Communication Quarterly, 91(1), 59-77. doi : https://doi.org/10.1177/0093650213497979

Beam, M. A., Child, J. T., Hutchens, M. J. et Hmielowski, J. D. (2017). Context collapse and privacy management: Diversity in Facebook friends increases online news reading and sharing. New Media \& Society, 20(7), 2296-2314. doi : https://doi.org/10.1177/1461444817714790

Bobadilla, J., Ortega, F., Hernando, A. et Gutiérrez, A. (2013). Recommender systems survey. Knowledge-based systems, (46), 109-132. doi : https://doi.org/10.1016/i.knosys.2013.03.012

Boczkowski, P. J. et Mitchelstein, E. (2013). The news gap: When the information preferences of the media and the public diverge. Cambridge, États-Unis : The MIT Press.

Bodó, B., Helberger, N., Eskens, S. et Möller, J. (2019). Interested in Diversity. Digital Journalism, 7(2), 206-229. doi : https://doi.org/10.1080/21670811.2018.1521292

Browet, A. (2014). Algorithms for community and role detection in networks (Thèse de doctorat en mathématiques appliquées), Université catholique de Louvain, Louvain-laNeuve, Belgique.

Bruns, A. (2019). Are filter bubbles real? Chichester, Royaume-Uni : Wiley Polity Press.

Bucher, T. (2017). The algorithmic imaginary: exploring the ordinary affects of Facebook algorithms. Information, 
Algorithmes de recommandation et culture technique : penser le dialogue entre éducation et design

Communication \& Society, 20(1), 30-44.

doi : https://doi.org/10.1080/1369118X.2016.1154086

Charbonnier, P. et Kreplak, Y. (2012). Savoirs écologiques.

Tracés. Revue de Sciences humaines, (22), 7-23.

doi : https://doi.org/10.4000/traces.5415

Chateauraynaud, F. (2006). Les Asymétries de prises. Des formes de pouvoir dans un monde en réseau. Paris, France : GSPR.

Chouteau, M., Escudie, M.-P., Forest, J. et Nguyen, C. (2015). La technique est-elle condamnée à entrer par effraction dans notre culture ? Phronesis, 4(2), 5-16.

doi : https://doi.org/10.7202/1033446ar

Citton, Y. (2014). Pour une écologie de l'attention. Paris, France : Éditions du Seuil.

Claussen, J., Peukert, C. et Sen, A. (2019). The Editor vs. The Algorithm: Returns to Data and Externalities in Online News [CESifo Working Paper No. 8012]. Repéré à : https://ssrn.com/abstract $=3518959$

Conover, M., Ratkiewicz, J., Francisco, M., Gonçalves, B., Flammini, A. et Menczer, F. (2011, juillet). Political Polarization on Twitter. Communication présentée à la $5^{\text {th }}$ International AAAI Conference on Weblogs and Social Media (ICWSM), Barcelone (Espagne).

De Certeau, M. (1990). L'invention du quotidien (tome 1 : Arts de faire). Paris, France : Gallimard.

De Noblet, J. (1981). Manifeste pour le développement de la Culture Technique. Culture Technique et changement de société. Culture Technique, (6), 11-47.

Del Vicario, M., Zollo, F., Caldarelli, G., Scala, A., et Quattrociocchi, W. (2017). Mapping social dynamics on Facebook: The Brexit debate. Social Networks, (50), 6-16. doi : https://doi.org/10.1016/j.socnet.2017.02.002

Dubois, E. et Blank, G. (2018). The echo chamber is overstated: The moderating effect of political interest and diverse media. Information, Communication \& Society, 21(5), 729-745. doi : https://doi.org/10.1080/1369118X.2018.1428656

Dylko, I., Dolgov, I., Hoffman, W., Eckhart, N., Molina, M. et Aaziz, O. (2017). The dark side of technology: An experimental 
Arnaud CLAES et al.

investigation of the influence of customizability technology on online political selective exposure. Computers in Human Behavior, (73), 181-190.

doi : https://doi.org/10.1016/j.chb.2017.03.031

Fastrez, P. et Philippette, T. (2017). Un modèle pour repenser l'éducation critique aux médias à l'ère du numérique. tic\&société, 11(1), 85-110. doi :

https://doi.org/10.4000/ticetsociete.2266

Fletcher, R. et Nielsen, R. K. (2018). Are people incidentally exposed to news on social media? A comparative analysis.

New Media \& Society, 20(7), 2450-2468.

doi : https://doi.org/10.1177/1461444817724170

Flaxman, S., Goel, S. et Rao, J. M. (2016). Filter Bubbles, Echo Chambers, and Online News Consumption. Public Opinion Quarterly, 80(Special Issue), 298-320.

doi : https://doi.org/10.1093/poq/nfw006

Garimella, K., De Francisci Morales, G., Gionis, A. et Mathioudakis, M. (2018, avril). Political Discourse on Social Media: Echo Chambers, Gatekeepers, and the Price of Bipartisanship. Communication présentée à la 2018 World Wide Web Conference, Lyon (France).

doi : https://doi.org/10.1145/3178876.3186139

Garrett, R. K. (2009). Politically Motivated Reinforcement Seeking: Reframing the Selective Exposure Debate. Journal of Communication, 59(4), 676-699.

doi : https://doi.org/10.1111/j.1460-2466.2009.01452.x

Garrett, R. K. (2013). Selective Exposure: New Methods and New Directions. Communication Methods and Measures, 7(3-4), 247-256. doi : https://doi.org/10.1080/19312458.2013.835796

Guattari, F. (2005). The three ecologies. London, États-Unis : Continuum.

Haim, M., Graefe, A. et Brosius, H.-B. (2018). Burst of the Filter Bubble? Digital Journalism, 6(3), 330-343.

doi : https://doi.org/10.1080/21670811.2017.1338145

Hamilton, K., Karahalios, K., Sandvig, C. et Eslami, M. (2014, Avril). A Path to Understanding the Effects of Algorithm

Awareness. Communication présentée au $\mathrm{CHI}$ '14 Human Factors in Computing Systems, Toronto (Canada).

doi : https://doi.org/10.1145/2559206.2578883 
Algorithmes de recommandation et culture technique : penser le dialogue entre éducation et design

Heaton, L., Millette, M. et Proulx, S. (2012). Médias sociaux : enjeux pour la communication. Québec, Canada : Presses de l'Université du Québec.

Holtz, D., Carterette, B., Chandar, P., Nazari, Z., Cramer, H. et Aral, S. (2020, juillet). The Engagement-Diversity Connection: Evidence from a Field Experiment on Spotify. Communication présentée au $21^{\text {st }} \mathrm{ACM}$ Conference on Economics and Computation, en ligne.

lyengar, S. et Hahn, K. S. (2009). Red Media, Blue Media: Evidence of Ideological Selectivity in Media Use. Journal of Communication, 59(1), 19-39.

doi : https://doi.org/10.1111/j.1460-2466.2008.01402.x

Jacobson, S., Myung, E. et Johnson, S. L. (2016). Open media or echo chamber: The use of links in audience discussions on the Facebook Pages of partisan news organizations. Information, Communication \& Society, 19(7), 875-891.

doi : https://doi.org/10.1080/1369118X.2015.1064461

Keller, R. (2013). Du singulier au sens large : intégrer analyse de discours et théorisation ancrée. Recherches Qualitatives, 15 (Hors Série), 416-434.

Kitchin, Rob (2016). Thinking Critically about and Researching Algorithms. Information, Communication, \& Society, 20(1), 14-29. doi :

https://doi.org/10.1080/1369118X.2016.1154087

Krafft, T. D., Gamer, M. et Zweig, K. A. (2019). What did you see? A study to measure personalization in Google's search engine. EPJ Data Science, 8(38).

doi : https://doi.org/10.1140/epjds/s13688-019-0217-5

Laurinaviciute, A. (2019). An algorithmic framework for avoiding filter bubbles (Mémoire de Master en Sciences des données). Université catholique de Louvain, Louvain-la-Neuve, Belgique.

Lee, D. et Hosanagar, K. (2019). How Do Recommender Systems Affect Sales Diversity? A Cross-Category Investigation via Randomized Field Experiment. Information Systems Research, 30(1), 239-259. doi : https://doi.org/10.1287/isre.2018.0800 


\section{Arnaud CLAES et al.}

Leskovec, J., Lang, K. J. et Mahoney, M. (2010, avril). Empirical comparison of algorithms for network community detection. Communication présentée au $19^{\text {th }}$ international conference on World wide web. Raleigh (États-Unis).

Litt, E. et Hargittai, E. (2016). The Imagined Audience on Social Network Sites. Social Media + Society, 2(1), 1-12. doi : https://doi.org/10.1177/2056305116633482

Messing, S. et Westwood, S. J. (2014). Selective Exposure in the Age of Social Media: Endorsements Trump Partisan Source Affiliation When Selecting News Online. Communication Research, 41(8), 1042-1063. doi : https://doi.org/10.1177/0093650212466406

Moeller, J. et Helberger, N. (2018). Beyond the filter bubble: Concepts, myths, evidence and issues for future debates (rapport). Amsterdam, Pays-Bas : Université d'Amsterdam.

Moumal, H. et Mottet, S. (2019). Ethics in Big Data: designing recommendation algorithms avoiding 'filter bubbles' (Mémoire de Master en Sciences Informatiques). Université catholique de Louvain, Louvain-la-Neuve, Belgique.

Munson, S. A. et Resnick, P. (2010, avril). Presenting diverse political opinions: How and how much. Communication présentée à la SIGCHI Conference on Human Factors in Computing Systems, Atlanta (États-Unis).

doi : https://doi.org/10.1145/1753326.1753543

Mutz, D. C. et Young, L. (2011). Communication and Public Opinion: Plus Ça Change? Public Opinion Quarterly, 75(5), 1018-1044. doi : https://doi.org/10.1093/pog/nfr052

Newman, N., Fletcher, R., Kalogeropoulos, A., Levy, D. A. L. et Nielsen, R. K. (2017). Reuters Institute Digital News Report 2017 [Rapport]. Repéré à : https://www.digitalnewsreport.org/.

Newman, N., Fletcher, R., Schulz, A., Simge, A. et Nielsen, R. K. (2020). Reuters Institute Digital News Report 2020 [Rapport]. Repéré à : https://www.digitalnewsreport.org/.

Nguyen, T. T., Hui, P.-M., Harper, F. M., Terveen, L. et Konstan, J. A. (2014, avril). Exploring the filter bubble: The effect of using recommender systems on content diversity. Communication présentée à la $23^{\text {rd }}$ International Conference on World Wide Web, Séoul (Corée du Sud). doi : https://doi.org/10.1145/2566486.2568012 
Algorithmes de recommandation et culture technique : penser le dialogue entre éducation et design

Ouellet, M., Mondoux, A. et Ménard, M. (2014). Médias sociaux, idéologie invisible et réel : pour une dialectique du concret. tic\&société, 8(1-2).

doi : https://doi.org/10.4000/ticetsociete.1391

Pariser, E. (2011). The filter bubble: what the Internet is hiding from you. Londres, Royaume-Uni : Penguin Books.

Paillé, P., \& Mucchielli, A. (2016). L'analyse qualitative en sciences humaines et sociales. Paris, France : Armand Colin.

Plantin, J.-C., Lagoze, C., Edwards, P. N. et Sandvig, C. (2018). Infrastructure studies meet platform studies in the age of Google and Facebook. New Media \& Society, 20(1), 293310. doi : https://doi.org/10.1177/1461444816661553

Powers, E. (2017). My News Feed is Filtered? Awareness of news personalization among college students. Digital Journalism, (5), 1315-1335.

doi : https://doi.org/10.1080/21670811.2017.1286943

Puschmann, C. (2019). Beyond the Bubble: Assessing the Diversity of Political Search Results. Digital Journalism, 7(6), 824-843. doi : https://doi.org/10.1080/21670811.2018.1539626

Quattrociocchi, W., Scala, A. et Sunstein, C. R. (2016). Echo Chambers on Facebook [SSRN].

Roth, C., Mazières, A. et Menezes, T. (2020). Tubes and bubbles topological confinement of YouTube recommendations. PLOS ONE, 15(4).

doi : https://doi.org/10.1371/journal.pone.0231703

Roqueplo, P. (1983). Penser la technique : pour une démocratie concrète. Paris, France : Éditions du Seuil.

Scharkow, M., Mangold, F., Stier, S. et Breuer, J. (2020, janvier). How social network sites and other online intermediaries increase exposure to news. Communication présentée à la National Academy of Sciences of the United States of America (PNAS). doi : https://doi.org/10.1073/pnas. 1918279117

Sears, D. O. et Freedman, J. L. (1967). Selective exposure to information: A critical review. Public Opinion Quarterly, 31(2), 194-213. doi : https://doi.org/10.1086/267513 
Arnaud CLAES et al.

Simondon, G. (2012). Du mode d'existence des objets techniques. Paris, France : Aubier.

Sinha, R. et Swearingen, K. (2002, Avril). The role of transparency in recommender systems. Communication présentée au CHl'02 Human factors in computing systems, Minneapolis (États-Unis).

doi : https://doi.org/10.1145/506443.506619

Star, S. L., et Ruhleder, K. (1996). Steps Toward an Ecology of Infrastructure: Design and Access for Large Information Spaces. Information Systems Research, 7(1), 111-134. doi : https://doi.org/10.1287/isre.7.1.111

Stiegler, B. (2016). Digital Studies-Organologie des savoirs et technologies de la connaissance. Limoges, France : FYP éditions.

Strauss, A. et Corbin, J. (1994). Grounded Theory Methodology: An Overview. Dans N.K. Denzin et Y.S. Lincoln (éd.), Handbook of Qualitative Research (Chapitre 17, p. 273-285). Thousand Oaks, États-Unis (CA) : SAGE publishing.

Sunstein, C. R. (2009). Republic.com 2.0. Princeton, ÉtatsUnis (NJ) : Princeton University Press.

Yang, T., Majó-Vázquez, S., Nielsen, R. K. et GonzálezBailón, S. (2020, octobre). Exposure to news grows less fragmented with an increase in mobile access. Communication présentée à la National Academy of Sciences of the United States of America (PNAS).

doi : https://doi.org/10.1073/pnas.2006089117

Yeung, K. (2017). 'Hypernudge': Big Data as a mode of regulation by design. Information, Communication \& Society, 20(1), 118-136.

doi : https://doi.org/10.1080/1369118X.2016.1186713

Zuiderveen Borgesius, F. J., Trilling, D., Möller, J., Bodó, B., de Vreese, C. H. et Helberger, N. (2016). Should we worry about filter bubbles? Internet Policy Review, 5(1), 1-16. 